gap $>\mathrm{g}:=$ SymmetricGroup $(4)$;

$\operatorname{Sym}\left(\left[\begin{array}{lll}1 & 4\end{array}\right]\right)$

gap> tbl:= CharacterTable ( $\mathrm{g}$ ); ; HasIrr ( tbl );

false

$05=$ total: $1 \begin{array}{rrrrr} & 4 & 13 & 14 & 4\end{array}$

gap> tblmod2:= CharacterTable( tbl, 2 );

BrauerTable( $\operatorname{Sym}([1 \ldots 4$ ] ), 2 )

1: 2242 gap> tblmod2 = CharacterTable ( tbl, 2 );

2: . 256 . true

Journal of Software for

01234 gap> libtbl:= CharacterTable( "M" );

$06=$ total: $1{ }^{4} 13144$ CharacterTable ( "M")

fail

gap> CharacterTable ( "Symmetric", 4 ); int a, b, c, t=11, 5, 3,0;

06 : BettiTally

CharacterTable ( "Sym(4)" )

gap> ComputedBrauerTables ( tbl );

$\begin{array}{lllll}0 & 1 & 2 & 3 & 4\end{array}$

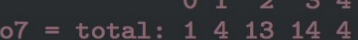

$0: 1 . .$.

[ BrauerTable( $\operatorname{Sym}([1 \ldots 4]), 2$ option(noprot);

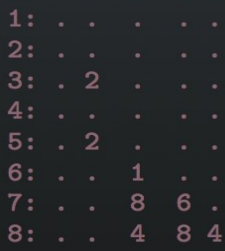

o7 : BettiTally

i8 : peek t1

timer $=1$;

ring $r 2=32003,(x, y, z), d p ;$

poly $f=\operatorname{imap}(r 1, f)$;

ideal $j=j a c o b(f)$;

$\operatorname{vdim}(\operatorname{std}(j))$;

$\Rightarrow 536$

$\operatorname{vdim}(\operatorname{std}(j+f))$;

$\Longrightarrow 195$

timer $=0 ; / /$ reset timer

$08=\operatorname{BettiTally}\{(0,\{0,0\}, 0) \Rightarrow 1\}$

$(1,\{2,2\}, 4) \Rightarrow 2$

$(1,\{3,3\}, 6) \Rightarrow 2$

$(2,\{3,7\}, 10) \Rightarrow 2$

$(2,\{4,4\}, 8) \Rightarrow 1$

$(2,\{4,5\}, 9) \Rightarrow 4$

$(2,\{5,4\}, 9) \Rightarrow 4$

$(2,\{7,3\}, 10) \Rightarrow 2$

$(3,\{4,7\}, 11) \Rightarrow 4$

$(3,\{5,5\}, 10) \Rightarrow 6$

Effective computitation of degree bounded minimal models of GCDAs

$(4,\{7,5\}, 12) \Rightarrow 2$

Victor Manero And Miguel Á. Marco BuzunÁriz 


\title{
Effective computation of degree bounded minimal models of GCDAs
}

\author{
Victor Manero And Miguel Á. Marco BuZunÁRiz
}

\begin{abstract}
Given a finitely presented graded commutative differential algebra (GCDA), we present a method to compute its minimal model up to a specified degree, together with a map that is a quasiisomorphism up to the given degree. The method works by adding generators one by one. It terminates if and only if the minimal model is finitely generated up to the given degree. A specific implementation of the method is given.

The method allows us to develop and implement two criteria for $i$-formality, one necessary and one sufficient. These criteria can be checked effectively, and have been able to determine the $i$-formality for every example that we have tested.
\end{abstract}

\section{INTRODUCTION.}

Definition 1. A graded commutative differential algebra (or GCDA) over a ring $\mathfrak{R}$ is a graded $\mathfrak{R}$-algebra $A=\bigoplus_{i=0}^{\infty} A_{i}$, together with an $\mathfrak{R}$-linear map $d_{A}: A \rightarrow A$ that satisfies the following conditions:

- $A_{i} A_{j} \subseteq A_{i+j}$.

- $d_{A}^{2}=0$.

- $d_{A}\left(A_{i}\right) \subseteq A_{i+1}$ for all $i \in \mathbb{N}$.

- $a b=(-1)^{i j} b a$ for all $a \in A_{i}, b \in A_{j}$.

- $d_{A}(a b)=d_{A}(a) b+(-1)^{i} a d_{A}(b)$ for all $a \in A_{i}, b \in A$.

From now on we will refer to a GCDA as a differential algebra, and when no confusion is possible we will denote the pair $\left(A, d_{A}\right)$ by $A$. To further simplify the notation, we will assume that the base ring $\mathfrak{R}$ is the field of rationals $\mathbb{Q}$, although the results will hold for more general fields. We will also assume that $A$ is connected, i.e., that $A_{0}=\mathbb{Q}$.

We will consider finitely presented differential algebras given by the following data:

- A finite set of homogeneous generators $\left\{a_{1}, \ldots, a_{n}\right\}$.

- For each generator $a_{j}$, a positive integer that will be its degree, which is denoted by $\left|a_{j}\right|$.

Manero is partially supported by MTM2017-85649-P (AEI/Feder, UE), UZCUD2019-CIE-02, and "Álgebra y Geometría" (Gobierno de Aragón/FEDER). Marco Buzunáriz is partially supported by MTM2016-76868-C2-2-P and Grupo "Investigación en Educación Matemática" of Gobierno de Aragón/Fondo Social Europeo.

MSC2010: 53-04, 55-04.

Keywords: graded commutative algebras, minimal model, formality, sagemath.

CommutativeDifferentialGradedAlgebras version 1.0 
- For each generator of degree $i$, its differential, which is a homogeneous, degree $i+1$ graded commutative polynomial in the generators.

- A finite set of homogeneous relations $\left\{R_{1}, \ldots, R_{m}\right\}$, which are graded commutative polynomials in the generators.

Given this data, $A$ is the quotient of the free $\mathbb{Q}$-algebra of graded commutative polynomials in $a_{1}, \ldots, a_{n}$ by the two-sided ideal $I$ generated by the relations $R_{1}, \ldots, R_{m}$. Choosing a monomial ordering, a Gröbner basis for $I$ determines a normal form (and hence, a unique representation) for the elements in $A$.

The notion of $i$-minimal differential algebra is well known in the literature (see for example [Suciu and Wang 2019]). We include the definition here for completeness.

Definition 2. A differential algebra $A$ is said to be $i$-minimal (in the sense of Sullivan) if it is freely generated as a graded commutative algebra by a collection of elements $\left\{a_{l}\right\}$ with $l \in J$, of $A$, for a wellordered index set $J$, such that $\left|a_{l}\right| \leq\left|a_{s}\right| \leq i$ if $l \leq s$ and the differential of a generator $a_{s}$ is expressed in terms of the $a_{l}$ 's with $l<s$.

Given differential algebras $A$ and $B$, a morphism of algebras

$$
\phi: A \rightarrow B
$$

is said to be a morphism of differential algebras if it preserves the degree and commutes with the differential. Those differential algebras are said to be $i$-quasi-isomorphic if there exists a morphism of differential algebras $\phi: A \rightarrow B$ such that $\phi^{*}: H^{j}(A) \rightarrow H^{j}(B)$ is an isomorphism for every $j \leq i$ and $\phi^{*}: H^{i+1}(A) \rightarrow H^{i+1}(B)$ is a monomorphism.

Definition 3. An $i$-minimal model of the differential algebra $\left(A, d_{A}\right)$ is an $i$-minimal differential algebra $\left(M, d_{M}\right)$ together with an $i$-quasi-isomorphism,

$$
\phi:\left(M, d_{M}\right) \rightarrow\left(A, d_{A}\right) .
$$

The existence and uniqueness of the minimal model of a connected differential algebra is guaranteed by the following result due to Halperin.

Theorem 1.1 [Morgan 1978; Sullivan 1977]. For any $i \geq 0$, any connected differential algebra A has an i-minimal model unique up to isomorphism.

Among the well-known examples of differential algebras is the de Rham complex $\left(\Omega^{*}(N), d_{N}\right)$ of differential forms on a manifold $N$. There is a construction that extends the idea of the de Rham algebra for general topological spaces, called the algebra of polynomial differential forms $A_{P L}$ (see, for instance, [Sullivan 1977]). An interesting well-known fact about this algebra is that its minimal model provides the rational homotopy groups of the space. Every differential algebra $\left(A, d_{A}\right)$ has associated to it another differential algebra, which is exactly the algebra given by its cohomology with zero differential, i.e., $H^{*}(A)$. An algebra whose minimal model is isomorphic to the minimal model of its cohomology algebra is called formal. Formality of de Rham algebras has been used to provide obstructions to the existence of certain geometrical structures on differentiable manifolds (see [Deligne et al. 1975], for example). 
2. EFFeCtive COMPUtATION OF THE Minimal MODEL. In this section we give a description of an algorithm to compute the $i$-minimal model of a connected differential algebra. It follows the usual approach in the literature, but is presented as an explicit algorithm.

We aim to construct a differential algebra $M$ that is an $i$-minimal model of $A$, together with a morphism of differential algebras $\phi: M \rightarrow A$ that is an $i$-quasi-isomorphism. In order to describe it, we need a set of generators as in Definition 2, and for each of them, its degree, differential and image under $\phi$. The method consists of adding generators sequentially, in such a way that we get the needed conditions. We will use the following notation:

- $x_{j}^{k}$ will denote a generator of $M$ of degree $k$ whose differential is zero.

- $y_{j}^{k}$ will denote a generator of $M$ of degree $k$ with nonzero differential.

Each time we add a new generator to $M$, we will use the diagram

$$
b_{j}^{k} \leftarrow y_{j}^{k} \rightarrow z_{j}^{k+1}
$$

to denote that we have added the generator $y_{j}^{k}$, with $d_{M}\left(y_{j}^{k}\right)=z_{j}^{k+1}$ and $\phi\left(y_{j}^{k}\right)=b_{j}^{k}$. The resulting algebra will be the free graded commutative algebra generated by the elements of the middle column, following the order in which they appear in the diagram. By abuse of notation, $M$ will denote the algebra freely generated by the generators found up to this point (that is, the meaning of $M$ will be updated after each step of the process).

2A. First step. We start by computing the smallest $k_{0}>0$ for which $H^{k_{0}}(A)$ is not trivial. Take a basis $\left[a_{0}^{k_{0}}\right], \ldots,\left[a_{l_{0}}^{k_{0}}\right]$ of $H^{k_{0}}(A)$. The first generators to add to $M$ are $x_{0}^{k_{0}}, \ldots, x_{l_{k_{0}}}^{k_{0}}$. That is, we start with the diagram

$$
\begin{gathered}
a_{1}^{k_{0}} \leftarrow x_{0}^{k_{0}} \rightarrow 0 \\
\vdots \\
a_{l_{k_{0}}}^{k_{0}} \leftarrow x_{l_{k_{0}}}^{k_{0}} \rightarrow 0
\end{gathered}
$$

At this point, we know that $\phi: M \rightarrow A$ induces an isomorphism $H^{j}(M) \rightarrow H^{j}(A)$ for all $j \leq k_{0}$.

2B. Increase degree. Assume that we have already found generators of degree up to $k-1$, such that the map $\phi: M \rightarrow A$ induces isomorphisms $\phi_{m}^{*}: H^{m}(M) \rightarrow H^{m}(A)$ for all $m \leq k-1$. In this step, we will add new generators to get also an isomorphism $\phi_{k}^{*}: H^{k}(M) \rightarrow H^{k}(A)$, without changing the lower degree cohomologies.

This step has two phases. In the first phase, which might need to be run iteratively, we will add generators of the form $y_{j}^{k-1}$ until the obtained map in $\phi_{k}^{*}$ is injective.

Once we have an injective map, we will add generators $x_{j}^{k}$, which will respect the lower degree differentials and the injectivity at degree $k$, until we get a surjective map.

2B1. Adding generators of the form $y_{j}^{k-1}$. Compute the $k$-th cohomology group $H^{k}(M)$ with the previously defined generators, and the induced map $\phi_{k}^{*}: H^{k}(M) \rightarrow H^{k}(A)$. If this map $\phi_{k}^{*}$ is not injective, 
take $\left[z_{0}^{k}\right], \ldots,\left[z_{l_{k-1}^{1}}^{k}\right]$ a basis of $\operatorname{Ker}\left(\phi_{k}^{*}\right)$. Now consider representatives $z_{0}^{k}, \ldots, z_{l_{k-1}^{1}}^{k} \in M$. Compute $c_{j}^{k}=\phi\left(z_{j}^{k}\right) \in A_{k}$. Since $\left[z_{j}^{k}\right]$ is in the kernel of the cohomology map, $c_{j}^{k}$ must correspond to a trivial cohomology class, that is, there must be $b_{j}^{k-1} \in A_{k-1}$ such that $d_{A}\left(b_{j}^{k-1}\right)=c_{j}^{k}$. So we add the generators

$$
\begin{gathered}
b_{0}^{k-1} \leftarrow y_{0}^{k-1} \rightarrow z_{0}^{k} \\
\vdots \\
b_{l_{k-1}^{k-1}} \leftarrow y_{l_{k-1}^{1}}^{k-1} \rightarrow z_{l_{k-1}^{k}}^{k}
\end{gathered}
$$

Note that, after adding these generators, new elements of $\operatorname{Ker}\left(\phi_{k}^{*}\right)$ could have been added. We can iterate this process, if required, by adding new generators

$$
\begin{aligned}
& b_{0}^{k-1} \leftarrow y_{0}^{k-1} \rightarrow z_{0}^{k} \\
& b_{l_{k-1}^{1}}^{k-1} \leftarrow y_{l_{k-1}^{1}}^{k-1} \quad \rightarrow \quad z_{l_{k-1}^{1}}^{k} \\
& b_{l_{k-1}^{1}+1}^{k-1} \leftarrow y_{l_{k-1}^{1}+1}^{k-1} \rightarrow z_{l_{k-1}^{1}+1}^{k} \\
& b_{l_{k-1}^{1}+l_{k-1}^{2}}^{k-1} \leftarrow y_{l_{k-1}^{1}+l_{k-1}^{2}}^{k-1} \rightarrow z_{l_{k-1}^{1}+l_{k-1}^{2}}^{k}
\end{aligned}
$$

until the map $\phi_{k}^{*}$ is injective.

2B2. Adding generators of the form $x_{n}^{k}$. If this map $\phi_{k}^{*}$ is not surjective, choose a basis

$$
\left[a_{1}^{k}\right], \ldots,\left[a_{j}^{k}\right],\left[a_{j+1}^{k}\right], \ldots,\left[a_{l_{k}}^{k}\right],
$$

of $H^{k}(A)$, where $\left[a_{j+1}^{k}\right], \ldots,\left[a_{l_{k}}^{k}\right]$ is a basis of $\operatorname{Im}\left(\phi_{k}^{*}\right)$. Add the new generators

$$
\begin{gathered}
a_{1}^{k} \leftarrow x_{1}^{k} \rightarrow 0 \\
\vdots \\
a_{j}^{k} \leftarrow x_{j}^{k} \rightarrow 0
\end{gathered}
$$

Repeat these two steps until $k=i$. Then we repeat step 2B1 one last time to get injectivity in $\phi_{i+1}^{*}$.

Example 2.1. Let $A$ be the algebra generated by six generators of degree $1 ; e_{1}, \ldots, e_{6}$, and one generator of degree $2 ; e_{7}$, with no relations, and where the differential is given by

$$
\begin{gathered}
d_{A}\left(e_{1}\right)=-e_{1} \wedge e_{6}, \quad d_{A}\left(e_{2}\right)=-e_{2} \wedge e_{6}, \quad d_{A}\left(e_{3}\right)=-e_{3} \wedge e_{6}, \\
d_{A}\left(e_{4}\right)=-e_{5} \wedge e_{6}, \quad d_{A}\left(e_{5}\right)=d_{A}\left(e_{6}\right)=d_{A}\left(e_{7}\right)=0 .
\end{gathered}
$$

We can compute the first cohomology groups

- $H^{1}(A)=\mathbb{Q}\left\langle e_{5}, e_{6}\right\rangle$,

- $H^{2}(A)=\mathbb{Q}\left\langle e_{4} \wedge e_{5}, e_{4} \wedge e_{6}, e_{7}\right\rangle$,

- $H^{3}(A)=\mathbb{Q}\left\langle e_{4} \wedge e_{5} \wedge e_{6}, e_{5} \wedge e_{7}, e_{6} \wedge e_{7}\right\rangle$,

where $\mathbb{Q}\left\langle E_{1}, \ldots, E_{n}\right\rangle$ denotes the vector subspace with basis $\left\{E_{1}, \ldots, E_{n}\right\}$. 
So we can start the method at degree 1 :

$$
\begin{aligned}
& e_{6} \leftarrow x_{0}^{1} \rightarrow 0 \\
& e_{5} \leftarrow x_{1}^{1} \rightarrow 0
\end{aligned}
$$

At this point, $H^{2}(M)$ would be generated by the class of $x_{0}^{1} \wedge x_{1}^{1}$. But the image by the induced map is $-e_{5} \wedge e_{6}$, which is trivial in cohomology, because it is the differential of $e_{4}$. So we have to add a new generator in order to make this cohomology class trivial:

$$
e_{4} \leftarrow y_{0}^{1} \rightarrow x_{0}^{1} \wedge x_{1}^{1}
$$

Now we get that $d_{M}\left(x_{0}^{1} \wedge y_{0}^{1}\right)=d_{M}\left(x_{1}^{1} \wedge y_{1}^{1}\right)=0$, so a basis of $H^{2}(M)$ is formed by the classes of $\left(x_{0}^{1} \wedge y_{0}^{1}, x_{1}^{1} \wedge y_{0}^{1}\right)$. Their images by $\phi$ are $e_{4} \wedge e_{6}$ and $e_{4} \wedge e_{5}$, which are two elements of the basis of $H^{2}(A)$ specified above. That is, we have an injective map at the second cohomology level. Proceed as described in step $2 \mathrm{~B} 2$; that is, add a new generator that will be mapped to the remaining element of the basis of $H^{2}(A)$ :

$$
e_{7} \leftarrow x_{0}^{2} \rightarrow 0
$$

Concerning degree 3, a basis for the cohomology of $M$ is given by the classes of $x_{0}^{1} \wedge x_{1}^{1} \wedge y_{0}^{1}, x_{0}^{1} \wedge x_{0}^{2}$, and $x_{1}^{1} \wedge x_{0}^{2}$. Since these elements map to the basis of $H^{3}(A)$, we already have an isomorphism in degree 3 . Up to degree 3 the minimal model of $A$ is

$$
M_{3}=\bigwedge\left(x_{0}^{1}, x_{1}^{1}, y_{0}^{1}, x_{0}^{2}\right) \text { with } d_{M}\left(y_{0}^{1}\right)=x_{0}^{1} \wedge x_{1}^{1},
$$

where $\bigwedge\left(x_{0}^{1}, x_{1}^{1}, y_{0}^{1}, x_{0}^{2}\right)$ denotes the commutative graded algebra freely generated by $\left\{x_{0}^{1}, x_{1}^{1}, y_{0}^{1}, x_{0}^{2}\right\}$, with their corresponding degrees. In fact, in this case we can check that the result obtained is a minimal model for $A$ in any degree.

We can summarize the whole process with the complete diagram

$$
\begin{aligned}
& e_{6} \leftarrow x_{0}^{1} \rightarrow 0 \\
& e_{5} \leftarrow x_{1}^{1} \rightarrow 0 \\
& e_{4} \leftarrow y_{0}^{1} \rightarrow x_{0}^{1} \wedge x_{1}^{1} \\
& e_{7} \leftarrow x_{0}^{2} \rightarrow 0
\end{aligned}
$$

\section{PROOF OF CORRECTNESS.}

Lemma 3.1. The algebra $M$ obtained after each step of the previous process is minimal.

Proof. It is free because we do not add any relation at any moment of the process. The generators are added in increasing order, and the differential of each generator is always either zero or expressed in terms of the previous generators.

Lemma 3.2. Let $\phi: M \rightarrow A$ be the map obtained after step $2 A$. The induced map $H^{k_{0}}(M) \rightarrow H^{k_{0}}(A)$ is an isomorphism. 
Proof. Since each $x_{j}^{k_{0}}$ has zero differential, and $M^{k_{0}-1}=0$, they produce a basis of the cohomology group $H^{k_{0}}(M)$. It is clear that the map gives a bijection with a basis of $H^{k_{0}}(A)$.

Lemma 3.3. Assume that before an iteration of the step 2B1 the maps $\phi_{m}^{*}$ are bijective for all $m<k$; then they are also bijective after adding each generator $y_{j}^{k-1}$.

Proof. For the groups $H^{m}(M)$ with $m<k-1$, both the space of cocycles and coboundaries remain untouched.

Consider the addition of a single generator $y_{l}^{k-1}$. Let $M_{k-1}$ be the space of homogeneous graded commutative polynomials of degree $k-1$ on the previous generators. By construction, $d_{M}\left(y_{l}^{k-1}\right) \notin$ $d_{M}\left(M_{k-1}\right)$, so

$$
\operatorname{dim}\left(d_{M}^{k-1}\left(\mathbb{Q} y_{l}^{k-1} \oplus M_{k-1}\right)\right)=1+\operatorname{dim}\left(d_{M}^{k-1}\left(M_{k-1}\right)\right) .
$$

This implies that

$$
\operatorname{Ker}\left(d_{M}^{k-1}\right)=\operatorname{Ker}\left(d_{M}^{k-1}\right) \cap M_{k-1} .
$$

Hence at this degree also both cocycles and coboundaries remain untouched.

Note that for certain algebras $A$, the $i$-minimal model is not finitely generated. For example, consider the exterior algebra on two generators $a_{0}, a_{1}$ of degree 1 , quotiented by the relation $a_{0} \wedge a_{1}=0$, and trivial differential. In this case, $H^{1}(A)=A^{1}=\mathbb{Q} a_{0} \oplus \mathbb{Q} a_{1}$, and for higher degrees, everything is trivial. Its minimal model $M$ should have two elements $x_{0}, x_{1}$ in degree 1 , that map to $a_{0}$ and $a_{1}$ respectively. But since $x_{0} \wedge x_{1}$ maps to an element that is trivial in homology, it should be a coboundary in $M$, that is, there must exist some $y_{0} \in M^{1}$ such that $d\left(y_{0}\right)=x_{0} \wedge x_{1}$. However, $x_{0} \wedge y_{0}$ is also a cocycle, so it must be also a coboundary: that is, there must exist some $y_{1} \in M^{1}$ such that $d\left(y_{1}\right)=x_{0} \wedge y_{0}$. Repeating this reasoning, we get that there must be an infinite sequence of elements $y_{0}, y_{1}, \ldots$, such that $d\left(y_{i+1}\right)=x_{0} \wedge y_{i}$. It is easy to prove by induction that they must be linearly independent, so $M^{1}$ must have infinite dimension.

In these cases, step 2B1 cannot terminate, since the output would require an infinite number of generators. However, when the $i$-minimal model is finitely generated, the method does finish and gives a correct result:

Lemma 3.4. If step $2 B 1$ terminates, the resulting map $\phi: M \rightarrow A$ is a (k-1)-quasi-isomorphism.

Proof. The step terminates only if the kernel of the induced map in degree $k$ is zero.

Lemma 3.5. Assume that before step $2 B 2$ the map $\phi: M \rightarrow A$ is a $(k-1)$-quasi-isomorphism. Then, after step 2B2, the new map $\phi: M \rightarrow A$ is also a (k-1)-quasi-isomorphism. Moreover the induced map $H^{k}(M) \rightarrow H^{k}(A)$ is an isomorphism.

Proof. For every $m<k$, the map $H^{m}(M) \rightarrow H^{m}(A)$ does not change by the addition of the generators $x_{1}^{k}, \ldots, x_{j}^{k}$ in degree $k$.

Consider the map $H^{k}(M) \rightarrow H^{k}(A)$. By the previous lemmas, it was injective before step 2B2. The effect of adding the generators $x_{1}^{k}, \ldots, x_{j}^{k}$ is precisely to extend the vector space $H^{k}(M)$ with the needed generators to fill $H^{k}(A)$. 
Joining these results, we get that, if the method terminates at degree $i$, we have obtained an $i$-minimal model for $A$.

4. APPLICATION TO FORMALITy CRITERIA. In this section we will see how the previous method can be useful to determine the formality of a differential algebra.

Definition 4. A differential algebra $A$ is said to be $i$-formal if its $i$-minimal model is $i$-quasi-isomorphic to its cohomology algebra $H^{*}(A)$. Analogously, $A$ is said to be formal if it is $i$-formal for every $i \in \mathbb{Z}^{+}$.

Remark 1. This definition is equivalent to the fact that the $i$-minimal model of $A$ is also an $i$-minimal model of $H^{*}(A)$.

The notion of $i$-formality can be found in the literature under this same name. However, some authors call it $k$-stage formality (see [Măcinic 2010]). It should be noted that there exists a different notion of $s$-formality, introduced by Fernández and Muñoz [2005].

We will now see two criteria (one necessary and one sufficient) for $i$-formality that can be computed making use of the method described before.

Necessary criterion: numerical invariants. Let $A$ be a differential algebra. By using the previous method, we can compute an $i$-minimal model $M_{A} \rightarrow A$. We can also compute a presentation of the cohomology algebra $H^{*}(A)$ up to degree $i+1$, and then its $i$-minimal model $M_{H} \rightarrow H^{*}(A)$. By the previous remark, $A$ is $i$-formal if and only if $M_{A}$ and $M_{H}$ are isomorphic.

Determining whether two presentations correspond to isomorphic algebras or not is, in general, a hard problem. Therefore we will use some numerical invariants that are related to the construction process described in Section 2, and hence can be computed. In particular, these invariants coincide with the number of generators that are added in each step of the algorithm. Let us now see that these numbers are, in fact, invariants under isomorphism.

Let $M$ be a minimal algebra obtained by the method in Section 2. Consider the corresponding cochain complex

$$
M^{0} \stackrel{d_{0}}{\longrightarrow} M^{1} \stackrel{d_{1}}{\longrightarrow} M^{2} \stackrel{d_{2}}{\longrightarrow} \cdots
$$

We will define recursively two families of linear subspaces (denoted $V_{j}^{i}$ and $W_{j}^{i}$ ) and a family of subalgebras (denoted by $N_{j}^{i}$ ):

- $V_{0}^{0}:=\{0\}$.

- $N_{j}^{i}$ is the subalgebra generated by all the vector spaces $V_{k}^{l}$ for $l<i$ or $l=i, k \leq j$.

- $N_{\infty}^{i}=\bigcup_{k=0}^{\infty} N_{k}^{i}$.

- $W_{0}^{i}:=\operatorname{Ker}\left(d_{i}\right) \cap N_{\infty}^{i-1}$ for $i>0$.

- $V_{0}^{i}:=\operatorname{Ker}\left(d_{i}\right)$.

- $W_{j+1}^{i}:=d_{i}^{-1}\left(N_{j}^{i}\right) \cap N_{j}^{i}$ for $i>0, j \geq 0$.

- $V_{j+1}^{i}:=d_{i}^{-1}\left(N_{j}^{i}\right)$ for $i>0, j \geq 0$. 
It is clear that these subalgebras and vector spaces must be preserved by isomorphism of differential algebras, since their definition only involves the algebra structure and the differential.

Now consider the numbers $v_{j}^{i}:=\operatorname{dim}\left(V_{j}^{i} / W_{j}^{i}\right)$. Let us now see that these numbers are related to the steps followed in the algorithm of Section 2.

Denote by $M_{0}^{k_{0}}$ the algebra obtained after step 2A of the algorithm, $M_{j}^{i}$ the algebra obtained after the $j$-th iteration of step 2B1 in degree $i$, and $M_{0}^{i}$ the algebra obtained after step 2B2 in degree $i$.

The following diagram summarizes the inclusion $N_{j}^{i} \hookrightarrow M$ :

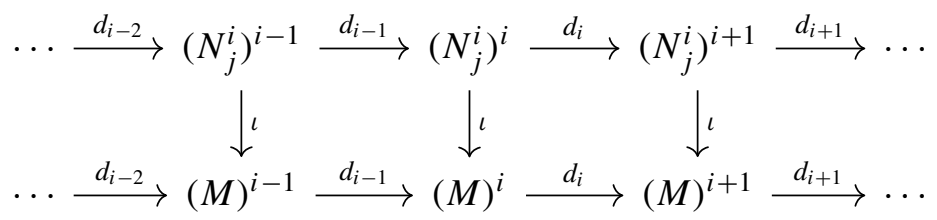

Lemma 4.1. The following properties hold

- $N_{j}^{i}=M_{j}^{i}$ for every $i, j$.

- The numerical invariant $v_{j}^{i}$ coincides with the number of generators added in the algorithm in the step that corresponds to $M_{j}^{i}$.

Proof. To prove that $N_{j}^{i}=M_{j}^{i}$, we proceed by induction on the steps of the algorithm. It is clear that for $k<k_{0}, M^{k}=\{0\}$, so all $V_{j}^{k}, W_{j}^{k}$ and $N_{j}^{k}$ are zero too. Let us see what happens at $k_{0}$.

By construction, $M^{k_{0}}$ admits a basis $x_{0}^{k_{0}}, \ldots, x_{l_{k_{0}}}^{k_{0}}, y_{0}^{k_{0}}, y_{1}^{k_{0}}, \ldots$ The differentials of these elements are of the form

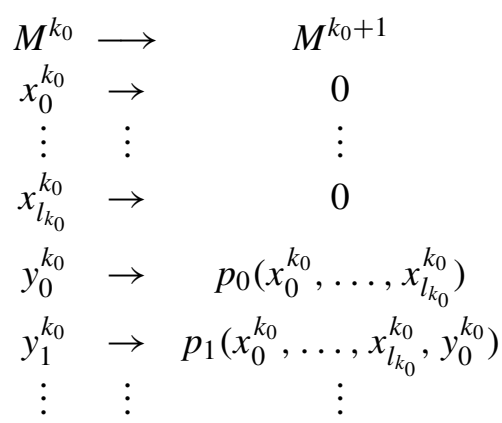

where the $p_{i}$ are linearly independent polynomials on the previous generators.

Then $V_{0}^{k_{0}}$ is the vector space generated by $x_{0}^{k_{0}}, \ldots, x_{l_{0}}^{k_{0}}$, and $N_{0}^{k_{0}}$ the subalgebra generated by them, which coincides with $M_{0}^{k_{0}}$. Also $W_{0}^{k_{0}}=\{0\}$, so $v_{0}^{k_{0}}$ coincides with the number of generators added in this step.

Now assume that $N_{j}^{i}$ coincides with $M_{j}^{i}$ for all previous steps of the algorithm, and we perform a new iteration of step 2B1. To simplify the notation, denote by $a$ the number of $y$ generators already added up to now. The difference between $M_{j}^{i}$ and $M_{j+1}^{i}$ lies in the addition of $b$ generators $y_{a+1}^{i}, \ldots, y_{a+b}^{i}$. The differential of all these generators lives in $M_{j}^{i}$. Since the differential of all the $y$ generators in degree $i$ are linearly independent, a basis of the space $V_{j+1}^{i}$ is obtained precisely by adding the generators 
$y_{a+1}^{i}, \ldots, y_{a+b}^{i}$ to a basis of $V_{j}^{i}$. Hence, $N_{j+1}^{i}$ coincides with $M_{j+1}^{i}$. Moreover, note that the generators $y_{a+1}^{i}, \ldots, y_{a+b}^{i}$ extend a basis of $W_{j+1}^{i}$ to a basis of $V_{j+1}^{i}$, so $v_{j+1}^{i}$ is indeed the number of generators added in this step.

Finally, let's see what happens at step 2B2. The generators $x_{0}^{i}, \ldots, x_{l_{i}}^{i}$ are chosen precisely to extend a basis of $\left(N_{\infty}^{i-1}\right)^{i}$ to a basis of $\left(N_{\infty}^{i-1}\right)^{i}+\operatorname{Ker}\left(d_{i}\right)$. That is, $V_{0}^{i}$ contains elements of $N_{\infty}^{i-1}$ plus the generators added in this step, so again $N_{0}^{i+1}=M_{0}^{i+1}$. As before, the generators $x_{0}^{i}, \ldots, x_{l_{i}}^{i}$ extend a basis of $W_{0}^{i}$ to a basis of $V_{0}^{i}$, so again the number of generators added coincides with $v_{0}^{i}$.

Corollary 1. If $A$ is formal, the numerical invariants $v_{j}^{i}$ of $M_{A}$ coincide with the ones of $M_{H}$.

Sufficient criterion. As before, let $A$ be a differential algebra and $M_{A}$ the $i$-minimal model obtained by the previous method. Consider the corresponding cohomology algebra $H^{*}(A) \cong H^{*}\left(M_{A}\right)$. We can compute its $i$-minimal model $M_{H}$. Assume that the numerical invariants of $M_{A}$ and $M_{H}$ coincide.

Consider the diagram followed to compute $M_{A}$ :

$$
\begin{aligned}
& a_{0}^{k_{0}} \leftarrow x_{0}^{k_{0}} \quad \rightarrow \quad 0 \\
& a_{l_{k_{0}}}^{k_{0}} \leftarrow x_{l_{k_{0}}}^{k_{0}} \quad \rightarrow \quad 0 \\
& b_{0}^{k-1} \leftarrow y_{0}^{k-1} \rightarrow z_{0}^{k} \\
& b_{l_{k-1}^{k}}^{k-1} \leftarrow y_{l_{k-1}^{1}}^{k-1} \quad \rightarrow \quad z_{l_{k-1}^{1}}^{k} \\
& b_{l_{k-1}^{1}+1}^{k-1} \leftarrow y_{l_{k-1}^{1}+1}^{k-1} \rightarrow z_{l_{k-1}^{1}+1}^{k} \\
& b_{l_{k-1}^{1}+l_{k-1}^{2}}^{k-1} \leftarrow y_{l_{k-1}^{1}+l_{k-1}^{2}}^{k-1} \rightarrow z_{l_{k-1}^{1}+l_{k-1}^{2}}^{k} \\
& a_{0}^{k} \leftarrow x_{0}^{k} \quad \rightarrow \quad 0 \\
& a_{l_{k}}^{k} \quad \leftarrow \quad x_{l_{k}}^{k} \quad \rightarrow \quad 0
\end{aligned}
$$

We define the morphism of algebras

$$
\begin{aligned}
\psi: M_{A} & \rightarrow H^{*}\left(M_{A}\right), \\
x_{j}^{i} & \rightarrow\left[x_{j}^{i}\right], \\
y_{j}^{i} & \rightarrow 0 .
\end{aligned}
$$

Definition 5. We will say that $M_{A}$ satisfies the $\psi$-condition if $\psi\left(z_{j}^{k}\right)=0$ for every $z_{j}^{k}$.

Note that the previous definition is equivalent to asking that $\psi$ is a morphism of differential algebras $\psi: M_{A} \rightarrow H^{*}\left(M_{A}\right)$. 
Lemma 4.2. If $M_{A}$ satisfies the $\psi$-condition and the numerical invariants of $M_{A}$ coincide with the ones of $M_{H}$, then $\psi$ is a i-quasi-isomorphism.

Proof. We will see this by proving the $i$-minimal model of $H^{*}\left(M_{A}\right)$ can be computed using the diagram

$$
\begin{aligned}
& {\left[x_{0}^{k_{0}}\right] \leftarrow x_{0}^{k_{0}} \rightarrow 0} \\
& {\left[x_{l_{k_{0}}}^{k_{0}}\right] \leftarrow x_{l_{k_{0}}}^{k_{0}} \quad \rightarrow \quad 0} \\
& 0 \leftarrow y_{0}^{k-1} \rightarrow z_{0}^{k} \\
& 0 \leftarrow y_{l_{k-1}^{1}}^{k-1} \rightarrow z_{l_{k-1}^{1}}^{k} \\
& 0 \leftarrow y_{l_{k-1}^{1}+1}^{k-1} \rightarrow z_{l_{k-1}^{1}+1}^{k} \\
& 0 \leftarrow y_{l_{k-1}^{1}+l_{k-1}^{2}}^{k-1} \rightarrow z_{l_{k-1}^{1}+l_{k-1}^{2}}^{k} \\
& \text { : } \\
& {\left[x_{0}^{k}\right] \leftarrow x_{0}^{k} \quad \rightarrow \quad 0} \\
& {\left[x_{l_{k}}^{k}\right] \leftarrow \begin{array}{c}
x_{l_{k}}^{k} \\
\vdots
\end{array}}
\end{aligned}
$$

The proof will be done by induction on the steps of the algorithm in Section 2. Note that the left part of the diagram is in fact the map $\psi$. That is, in this case, $\psi$ will play the role of $\phi$.

In step 2A we have to choose a basis of the first nontrivial cohomology group of $H^{*}\left(M_{A}\right)$. Since $H^{*}\left(M_{A}\right)$ is itself the cohomology algebra of $M_{A}$, its cohomology is isomorphic to itself. So we can choose $\left[x_{0}^{k_{0}}\right], \ldots,\left[x_{l_{0}}^{k_{0}}\right]$ as the basis of its first nonzero cohomology group. Hence, we can start the construction of the minimal model of $H^{*}\left(M_{A}\right)$ with the diagram

$$
\begin{gathered}
{\left[x_{0}^{k_{0}}\right] \leftarrow x_{0}^{k_{0}} \rightarrow 0} \\
\vdots \\
{\left[x_{l_{k_{0}}}^{k_{0}}\right] \leftarrow x_{l_{k_{0}}}^{k_{0}} \rightarrow 0}
\end{gathered}
$$

Now, for each iteration of step 2B1, let us assume as induction hypothesis that the diagram used up to this step coincides with diagram (2). To proceed with the iteration of the step, we need to choose a basis of $\operatorname{Ker}\left(\psi_{k}^{*}\right)$. Since the numerical invariants coincide, the dimension of this basis has to coincide with the number of generators added in this step of the construction of $M_{A}$. The cohomology classes of $z_{0}^{k}, \ldots, z_{l_{k-1}^{1}}^{k}$ do live in $\operatorname{Ker}\left(\psi_{k}^{*}\right)$ because of the $\psi$-condition. And they are linearly independent on the cohomology of $M_{A}$ because of the induction hypothesis (the cohomology of $M_{A}$ up to this point has to coincide with the one of $M_{H}$ ). So the same $z_{0}^{k}, \ldots, z_{l_{k-1}^{1}}^{k}$ that were used for constructing $M_{A}$ can be chosen to construct $M_{H}$. 
For the left part of the diagram, we have to choose preimages by the differential of $\psi\left(z_{0}^{k}\right), \ldots, \psi\left(z_{l_{k-1}^{1}}^{k}\right)$. Since all these elements are zero, we can choose zero as its preimages, so we can extend the diagram with

$$
\begin{gathered}
0 \leftarrow y_{0}^{k-1} \rightarrow z_{0}^{k} \\
\vdots \\
0 \leftarrow y_{l_{k-1}^{k-1}}^{k-1} \rightarrow z_{l_{k-1}^{1}}^{k}
\end{gathered}
$$

For the step 2B2, again the condition on the numerical invariants tells us that we have to add the same number of generators to the diagram. As before, the classes $\left[x_{1}^{k}\right], \ldots,\left[x_{j}^{k}\right]$ are linearly independent, in $H^{k}\left(M_{A}\right)$, and are not in the image of the previously added generators by $\psi$, so they are a suitable choice for this step.

Thanks to the previous lemma, given a GCDA $A$, we can try to decide the $i$-formality by the following steps:

- Compute a presentation of $M_{A}$, the $i$-minimal model of $A$. During the construction we get the numerical invariants of $M_{A}$.

- Compute a presentation of $H^{*}\left(M_{A}\right)$ up to degree $i+1$.

- Compute a presentation of $M_{H}$, the $i$-minimal model of $H^{*}\left(M_{A}\right)$. During the construction we get the numerical invariants of $M_{H}$.

- If the numerical invariants of $M_{A}$ and $M_{H}$ do not coincide, $A$ is not $i$-formal.

- If the numerical invariants do coincide, check if the presentation of $M_{A}$ satisfies the $\psi$-condition. If it does, $A$ is $i$-formal.

In principle, it could happen that the numerical invariants coincide but the presentation of $M_{A}$ does not satisfy the $\psi$-condition. If that happens we can not determine the $i$-formality in this way. However, we have not found any such example. That is, in every case we have tried, the method above allows us to determine if the algebra is $i$-formal or not. This motivates the following questions:

Question 1. Is the equality of numerical invariants up to degree $i$ a sufficient condition for $i$-formality? Question 2. Is the $\psi$-condition up to degree $i$ necessary for $i$-formality?

5. IMPLEMENTATION AND EXAMPLES. We present an implementation of the previous algorithms in [SageMath]. The computation of the minimal models and cohomology algebras have been included since version 8.8. The formality criteria were merged in version 9.0.

We now illustrate our implementation with an example.

\section{Examples of computations of minimal models with SageMath.}

Example 5.1. In SageMath we can define the differential algebra in Example 2.1 as follows:

sage: A.<e1, e2 e e3, e4, e5, e6, e7> = GradedCommutativeAlgebra(QQ,

degrees $=[1,1,1,1,1,1,2]$ )

sage: $B=A . c d g \_a l g e b r a(\{e 1:-e 1 * e 6, e 2:-e 2 * e 6, e 3:-e 3 * e 6, e 4:-e 5 * e 6\})$ 
Its 4-minimal model can be computed by

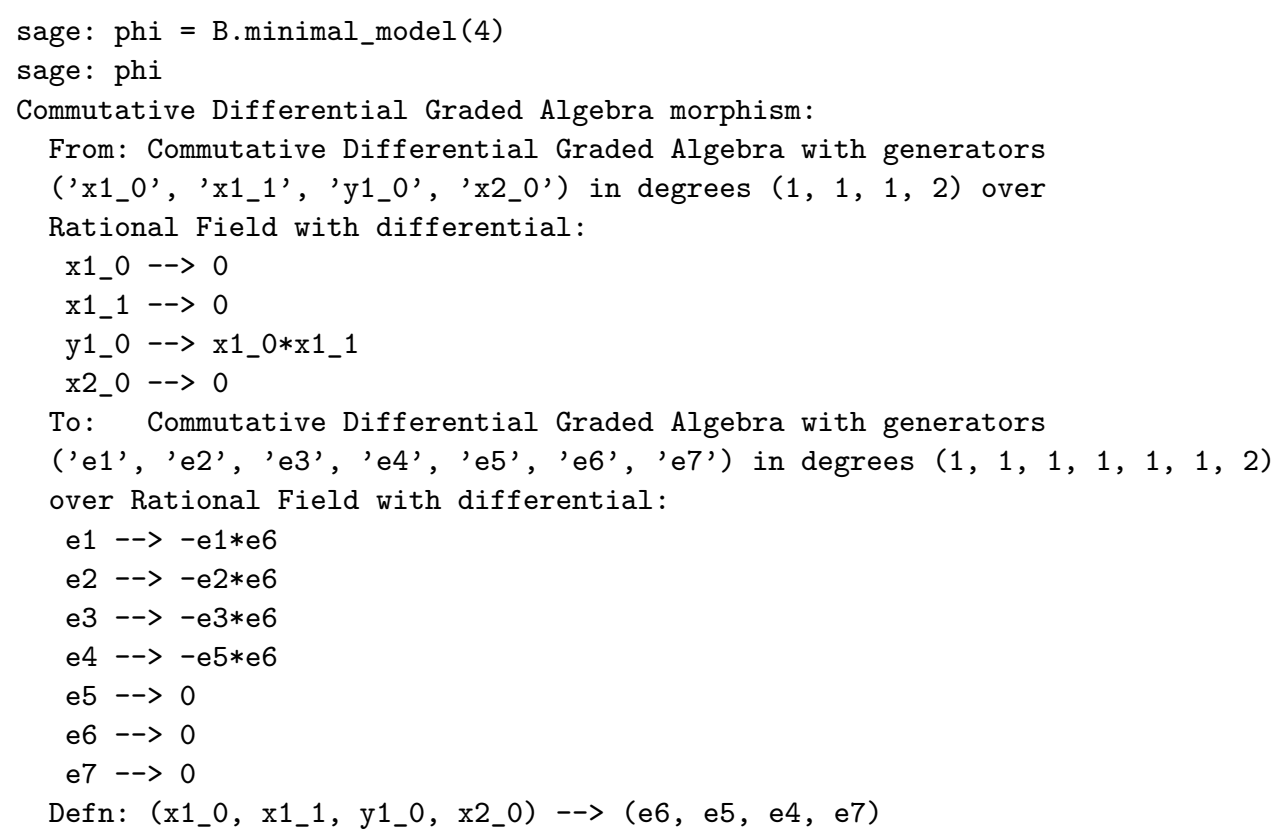

Notice that the result is given as a differential algebra morphism from the $i$-minimal model to the algebra given as input. That is, we get not only an abstract description of the $i$-minimal model, but also an explicit $i$-quasi-isomorphism. We can get the $i$-minimal model itself as the domain of the morphism:

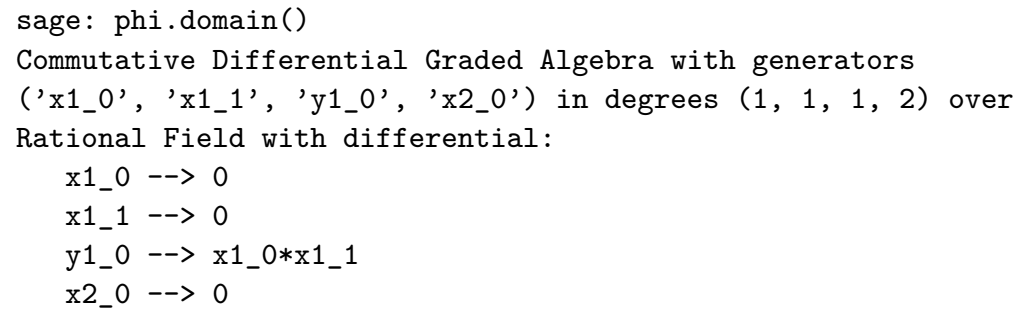

Note that here we see the right part of the diagram (1), whereas in the line

Defn: (x1_0, x1_1, y1_0, x2_0) --> (e6, e5, e4, e7)

we see the left part.

Example 5.2. We can also work with nonfree algebras. They must be introduced as the quotient of a free algebra by a bilateral ideal. For instance, the cohomology algebra of $\mathbb{S}^{2} \vee \mathbb{S}^{3}$ has only elements in degrees 2 and 3 .

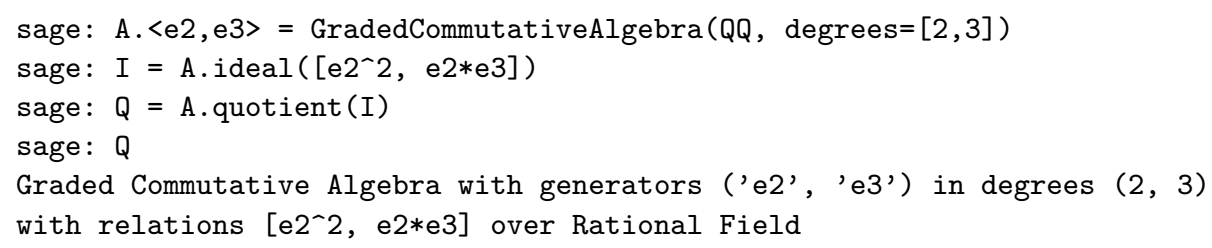


We can check that this algebra only has elements in degrees 2 and 3 :

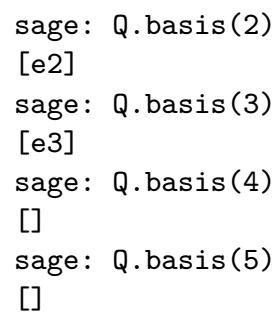

Now we define its corresponding GCDA with trivial differential, and compute its 6-minimal model.

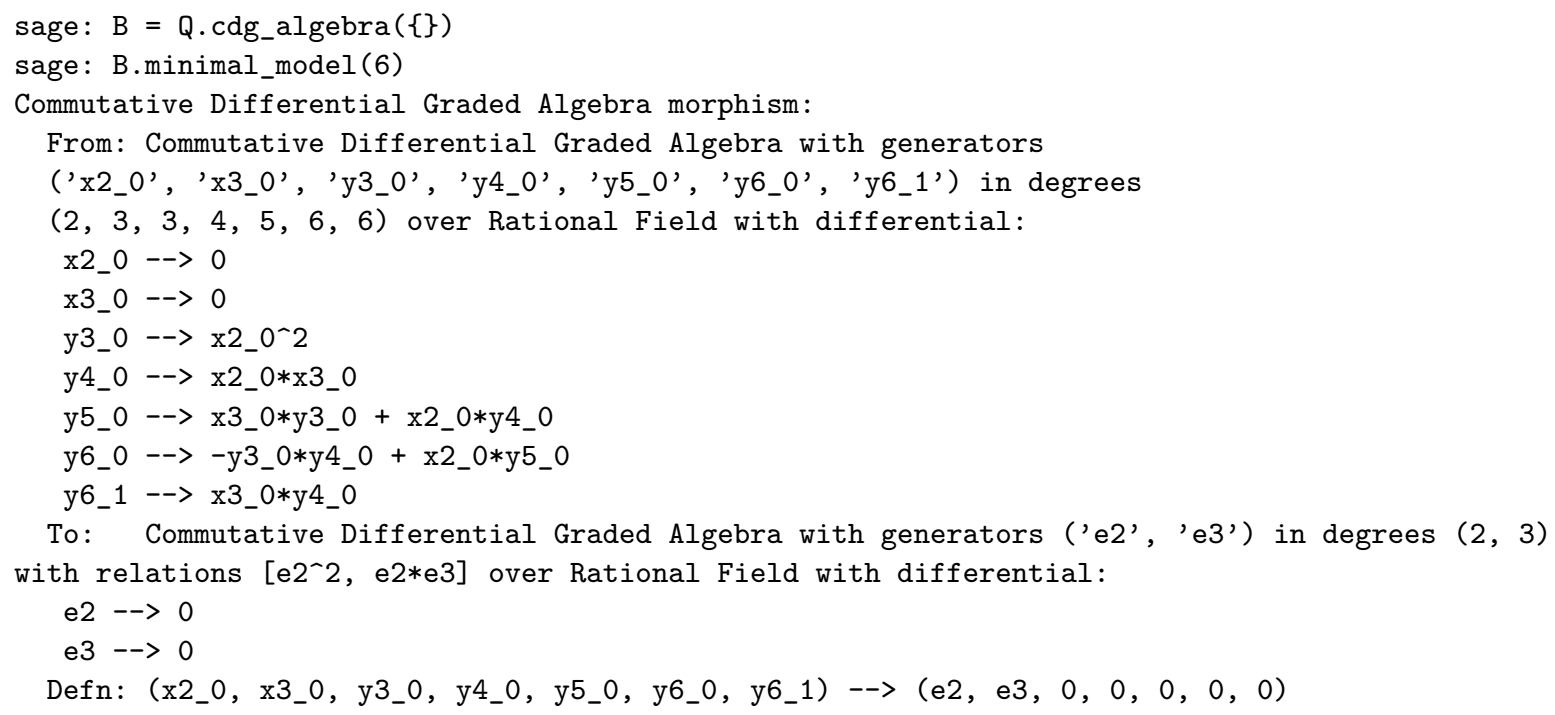

Examples of formality criteria with SageMath. Bock [2009] studied the formality of solvmanifolds up to dimension 6. In the following we show some examples that were not covered there.

Example 5.3. The algebra $G_{5.14}^{0}$ in [Bock 2009] is not formal (in fact, not even 2-formal):

sage: $A .\langle x 1, x 2, x 3, x 4, x 5\rangle=$ GradedCommutativeAlgebra(QQ)

sage: $\mathrm{B}=\mathrm{A} . \mathrm{cdg}$ _algebra $(\{\mathrm{x} 1:-\mathrm{x} 2 * \mathrm{x} 5, \mathrm{x} 4: \mathrm{x} 3 * \mathrm{x} 5, \mathrm{x} 3:-\mathrm{x} 4 * \mathrm{x} 5\})$

sage: B.is_formal (2)

False

Indeed, we can look at the 3-minimal model:

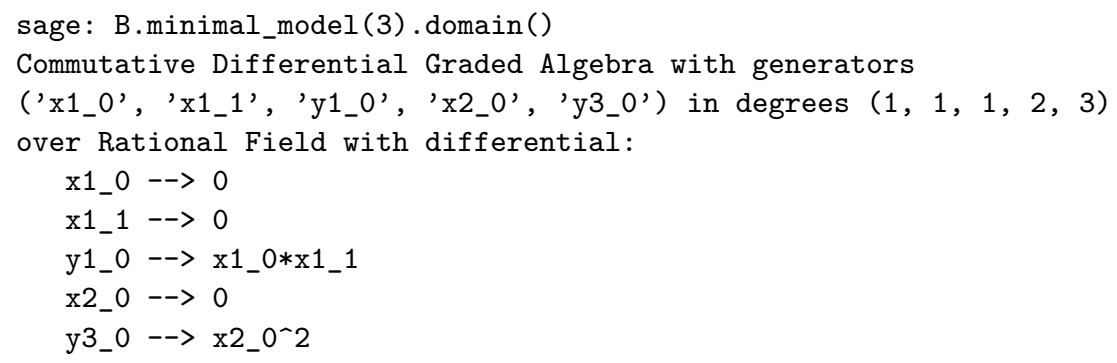


We can see that the nonzero numerical invariants are $v_{1}^{1}=1, v_{0}^{2}=1$ and $v_{1}^{3}=1$.

If we try to compute the 2-minimal model of the cohomology algebra, we get:

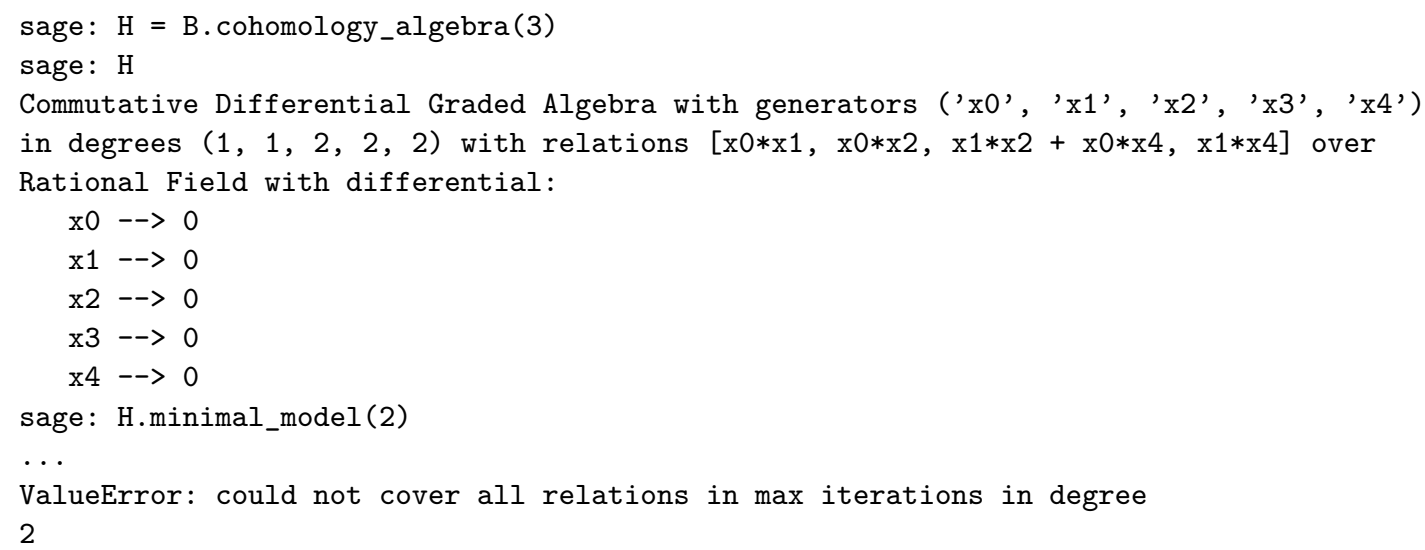

This means that the algorithm did not finish after three iterations of step 2B1 in degree 1 ( 3 is the default value to decide to give up). This implies that $M_{H}$ has more than three nonzero numerical invariants $v_{1}^{1}, v_{2}^{1}, v_{3}^{1}$ (in fact, it can be checked that for $M_{H}$, the first numerical invariants are $v_{0}^{1}=2, v_{1}^{1}=1, v_{2}^{1}=2$ and $v_{3}^{1}=3$ ), and hence it cannot be isomorphic to $M_{A}$.

Example 5.4. The case $G_{5.35}^{-2,0}$ in [Bock 2009] is 6-formal:

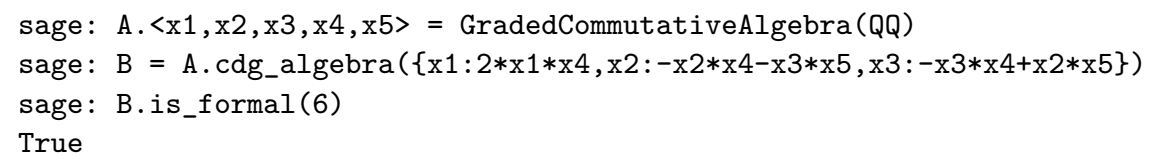

We can actually see that it is indeed formal. Since the algebra is generated by five generators of degree 1 , it is trivial beyond degree 5 . We can see that its 5-minimal model is also trivial beyond degree 5 (moreover, it coincides with its cohomology algebra):

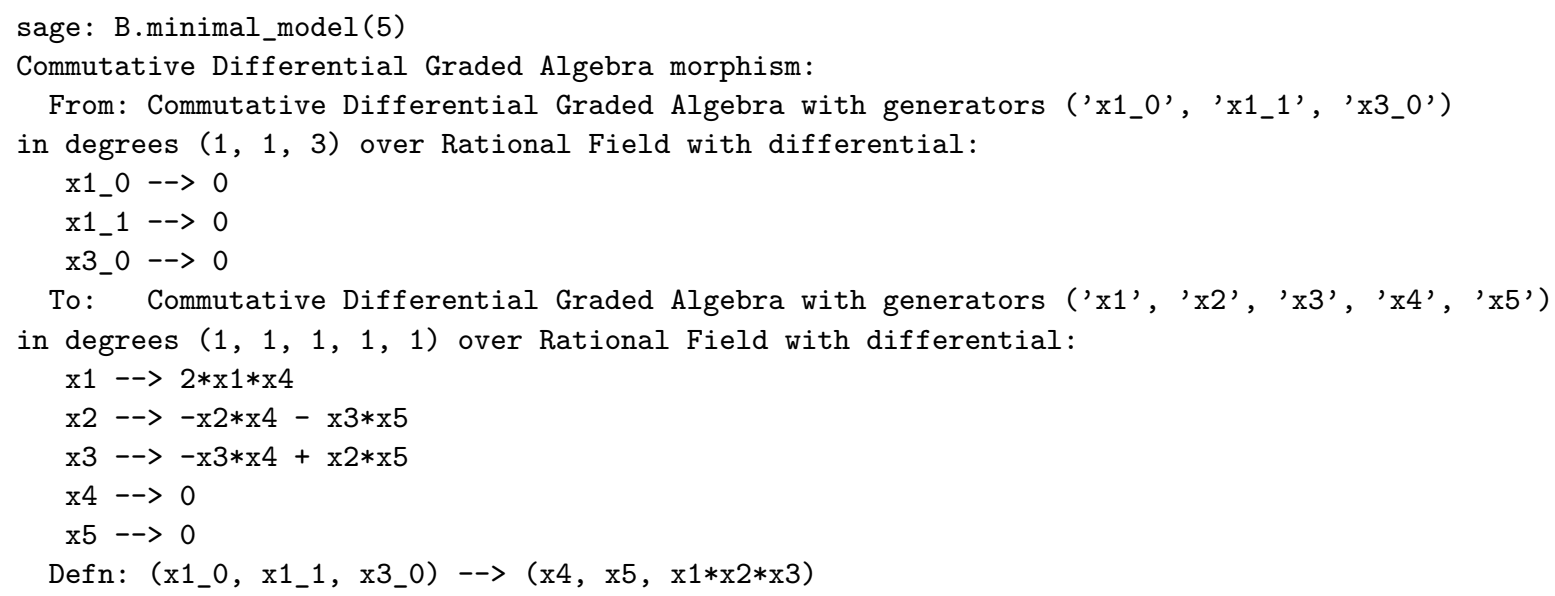

So the 5-minimal model is in fact the minimal model. Observe that it is isomorphic to the cohomology algebra. 
SUPPLEMENT. Version 1.0 of CommutativeDifferentialGradedAlgebras. is contained in the online supplement.

\section{REFERENCES.}

[Bock 2009] C. Bock, On formality and solvmanifolds, Ph.D. thesis, Universität zu Köln, 2009, available at https://tinyurl.com/ bock-thesis-pdf.

[Deligne et al. 1975] P. Deligne, P. Griffiths, J. Morgan, and D. Sullivan, "Real homotopy theory of Kähler manifolds", Invent. Math. 29:3 (1975), 245-274. MR Zbl

[Fernández and Muñoz 2005] M. Fernández and V. Muñoz, "Formality of Donaldson submanifolds", Math. Z. 250:1 (2005), 149-175. MR Zbl

[Morgan 1978] J. W. Morgan, "The algebraic topology of smooth algebraic varieties", Inst. Hautes Études Sci. Publ. Math. 48 (1978), 137-204. MR Zbl

[Măcinic 2010] A. D. Măcinic, "Cohomology rings and formality properties of nilpotent groups", J. Pure Appl. Algebra 214:10 (2010), 1818-1826. MR Zbl

[SageMath] The Sage Developers, "Sage Mathematics Software (Version 8.6.0)", available at http://www.sagemath.org.

[Suciu and Wang 2019] A. I. Suciu and H. Wang, "Formality properties of finitely generated groups and Lie algebras", Forum Math. 31:4 (2019), 867-905. MR Zbl

[Sullivan 1977] D. Sullivan, "Infinitesimal computations in topology", Inst. Hautes Études Sci. Publ. Math. 47 (1977), $269-331$. MR Zbl

\section{VICTOR MANERO:}

vmanero@unizar.es

Departamento de matemáticas - I.U.M.A, Universidad de Zaragoza, Huesca, Spain

Miguel Á. Marco BuZunÁRIz:

mmarco@unizar.es

Departamento de matemáticas - I.U.M.A., Universidad de Zaragoza, Zaragoza, Spain 